\title{
Visualisation of penile structures of laboratory rabbit: ultrasound, histology, and micro-CT
}

\author{
Mikhail Kogan ${ }^{1}$, Igor Popov ${ }^{1,2,}{ }^{*}$, Boris Mitrin ${ }^{2}$, Ilya Popov ${ }^{1,2}$, Evgeniy Sadyrin ${ }^{2}$, Dmitry \\ Pasetchnik $^{2}$, Alexey Ermakov ${ }^{2}$, Natia Ugrekhelidze $^{2}$, and Natalia Kulikova ${ }^{2}$ \\ ${ }^{1}$ Rostov State Medical University, 344022, 29, Nahichevansky st., Rostov-on-Don, Russia \\ ${ }^{2}$ Don State Technical University, 344003, 1, Gagarin Square, Rostov-on-Don, Russia
}

\begin{abstract}
Current imaging methods in pre-clinical and animal model studies of penile disease are limited because of the small penis of a rat standard laboratory animal used in this type of research. Routine visualization methods of surface and inner structures of the penis used in clinical medicine are not suitable in experimental animal researches. The only method available for these purposes is histopathological analysis, which does not provide complex view on penile structures due to twodimensional imaging per slide. In the pre study we evaluated and compared capability of ultrasound, histopathological and micro-computed tomography imaging methods to visualize anatomical structures of laboratory rabbit penis. Ultrasound provides limited imaging in comparison with other methods. However, it could be useful in assessment rough structural alterations of tunica albuginea and corpora cavernosa as a secondary and supportive method. Micro computed tomography provides nearly the same image quality as histopathological analysis. Moreover, it gives a complex view on the whole penile sample due to three-dimensional reconstruction. This fact gives reason to use this method as a basic one in the pre-clinical trials and animal model studies.
\end{abstract}

\section{Introduction}

Visualization plays a critical role in diagnosis of penile diseases of human and animals. Histopathological analysis remains the 'gold standard' in diagnostics of a wide range of diseases. The main reason for this is quality of image acquisition, which is possible due to the preservation of tissue architecture while histological slide preparation. Moreover, it is possible to use various stains to reveal different tissues and cellular components [1]. The most common one is Hematoxylin-Eosin (H\&E) staining, which has been used by pathologists for over a hundred years [2]. In most cases, histopathological analysis of penile bioptates is not suitable as it brings additional damage for penile tissue of patients and can induce secondary structure alterations [3]. For this purpose, physicians can use a wide range of other non-invasive diagnostic methods including ultrasound (US), magnetic resonance and computer tomography (CT) [4-6].

\footnotetext{
* Corresponding author: doc.igor.popov@gmail.com
} 
Visualization also takes a crucial part in pre-clinical trials of novel treatment methods of penile diseases and fundamental animal model studies. In most of these types of researches, the only imaging method available is histopathological analysis because of the relatively small size of laboratory animal penis. The main limitation of this kind of visualization is a lack of complex view on structures of taken samples as one histopathological slide provides only two-dimensional (2D) picture. This contributes to an inability to assess multiple structural alterations of penile tissues at once, such as the topical occurrence of fibrosis throughout corpora cavernosa in organic erectile dysfunction $[7,8]$, length of urethral constriction in urethral strictures [9] and tumor progression in penile cancer [3]. So, at the moment we cannot fully extrapolate results of animal model studies into clinical reality, as we cannot compare two-dimensional data of histological assessment of modelled penile disease progression of laboratory animal with results of three-dimensional (3D) visualization of human penile structures by aforementioned diagnostic methods [4-6].

We suggest that novel approach for visualization of laboratory animal penile structure by X-ray micro computed tomography (micro-CT) will increase the value of pre-clinical trials and experimental researches and especially their capability to be compared with clinical researches on human [10]. Micro-CT is a non-destructive imaging method enabling $3 \mathrm{D}$ visualization of surface and inner structure of any biological sample at a resolution comparable to light microscopy [11]. In this study we evaluated and compared capability of US, histopathological and micro-CT imaging methods to visualize anatomical structures of the laboratory rabbit penis.

\section{Material and Methods}

\subsection{Experimental design}

The experiment was carried out in accordance with the «Guide for the Care and Use of Laboratory Animals» and was approved by the ethics committee of DSTU, Rostov-on-Don, Russia (protocol number 67-43-1).

Penile US was conducted on 5 males $24.3 \pm 0.8$ weeks old white New Zealand rabbits using MINDRAY DC-7 («SHENZEN MINDRAY», Bio-Medical Electronics Co. Ltd., China). After that, all of laboratory animals underwent bilateral orchidectomy and penectomy following reconstruction of urethra at one time. Penises were fixed overnight in $10 \%$ neutral buffered formalin.

\subsection{Sample preparation}

The fixed tissue samples were gradually dehydrated in ethanol for hour in each solution $(50 \%, 70 \%, 80 \%, 90 \%, 96 \%, 100 \%)$ with hour pause between them. After that samples were stained in $1 \%$ wt I2 solution in absolute ethanol for 14 hours. The final step was washing in $100 \%$ ethanol following placing samples into plastic containers filled with $100 \%$ ethanol [12]. Samples were stored at $5^{\circ} \mathrm{C}$.

\subsection{Micro-CT Image Acquisition and Reconstruction}

Scans were acquired using the Xradia Versa 520 unit (Carl Zeiss X-ray Microscopy, Inc., USA). All samples were mounted on the sample holder in the plastic tubes filled with $100 \%$ ethanol. The X-ray source was set at $60 \mathrm{kV}$ and $4.5 \mathrm{~W}$ with a LE2 filter. Acquisition parameters used for scanning each sample: $0.4 \mathrm{X}$ magnification objective, $1.5 \mathrm{~s}$ exposure time, $50 \mu \mathrm{m}$ voxel size. For each sample, 1601 projection images were taken, the rotation 
was $360^{\circ}$. X-ray projections were afterwards reconstructed using XRMReconstructor 12.0.8086.19558 software (Carl Zeiss X-ray Microscopy Inc., USA) with automatically adjusted center shift values, $\sigma=0.5$ Gauss blurring filter, and standard beam hardening correction $=0.05$.

After data acquisition, the images were exported in DICOM format for volume rendering in VGSTUDIO MAX 3.4 software (Volume Graphics GmbH, Heidelberg, Germany). The imported DICOM files were rendered in Volume renderer (Scatter HQ).

\subsection{Histopathological analysis}

The samples were cut in the projection of region of interest corresponding to the micro-CT data and embedded in paraffin for further staining with H\&E. The slices obtained from the paraffin blocks were subsequently imaged by transmitted light microscopy and scanned using a histological slide scanner Aperio2 (Leica, Germany). Images were analyzed using QuPath [13].

\section{Results}

\subsection{Postoperative observation and animal care}

No lethal cases were registered. After the experiment, all laboratory animals underwent daily veterinary examination for the diseases presence and pain syndrome and obtained necessary treatment. The final examination was carried out and all animals without clinical signs of any disease were sent to the Educational Agricultural Center of DSTU after two weeks.[19]

\subsection{US imaging}

Laboratory animals were placed on table in a supine position during penile US examinations. The penile structures were identified by different echogenicity. However, the orientation was relatively difficult due to lack of the echogenicity of corpus spongiosum (Fig. 1).

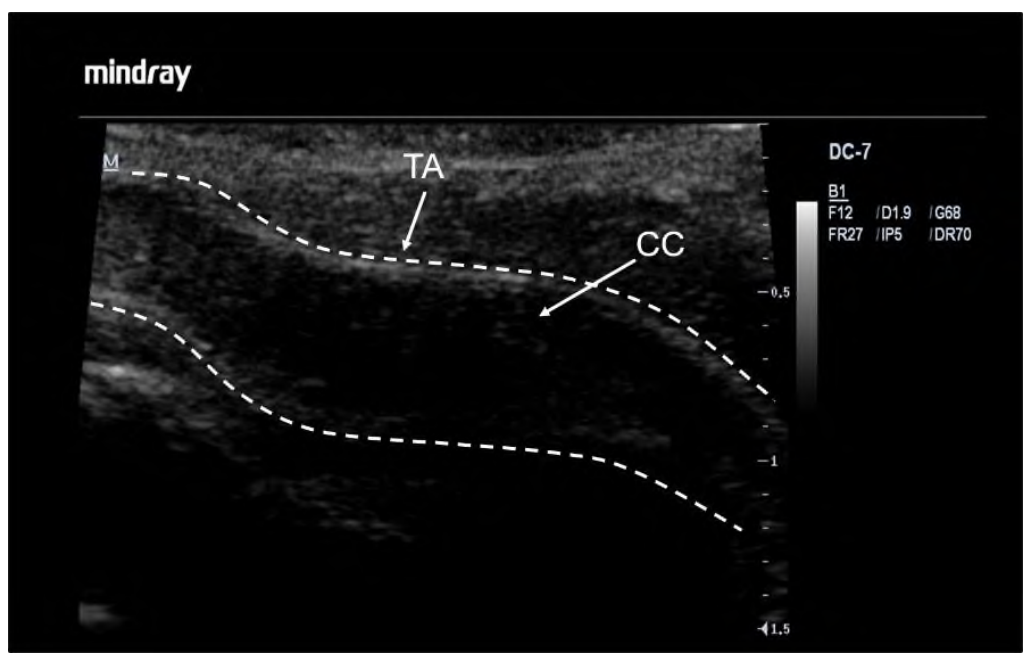


Fig. 1. Sonogram of laboratory rabbit penis. Penile structures are poorly contrasted, there is no possibility to define urethra. TA - tunica albuginea; $\mathrm{CC}$ - corpora cavernosa.

For better US visualization polyurethane feline urethral catheter with outside diameter $1.2 \mathrm{~mm}$ was inserted into the urethra. The thickness and high density of the catheter added echogenicity to the urethral wall, which made the orientation much easier (Fig. 2).

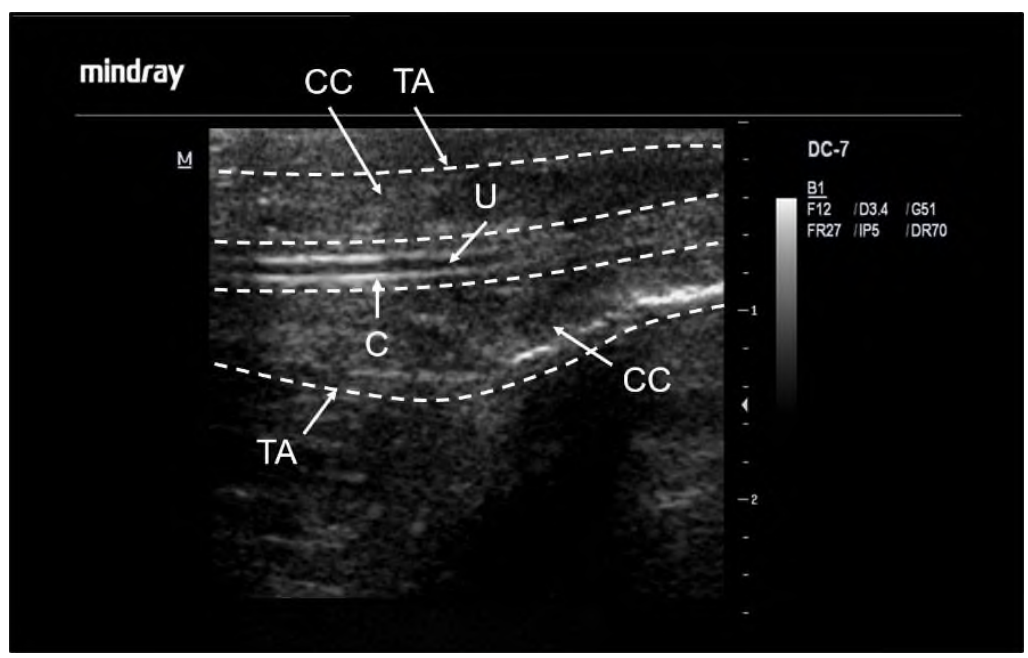

Fig. 2. Sonogram of laboratory rabbit penis with the inserted urethral catheter. TA - tunica albuginea; $\mathrm{CC}$ - corpus cavernosum; $\mathrm{C}$ - catheter; $\mathrm{U}$ - urethra.

\subsection{Micro-CT imaging}

Five 3D models of rabbit penises were acquired by micro-CT and following virtual reconstruction. Frontal and sagittal sections were made to visualize inner structures of penises, which were identified according to the micro-CT data from other animals and CT data from human $[5,12,13]$. Micro-CT allowed to visualize the whole penis at a time. Two corpora cavernosa are separated from each other but not completely along the entire length of the penis due to inconsistency of the septum. Intracavernosal pillars seem to be all over the inner surface of the penis, but they are not represented in the basis of crus penis (Fig. 3).

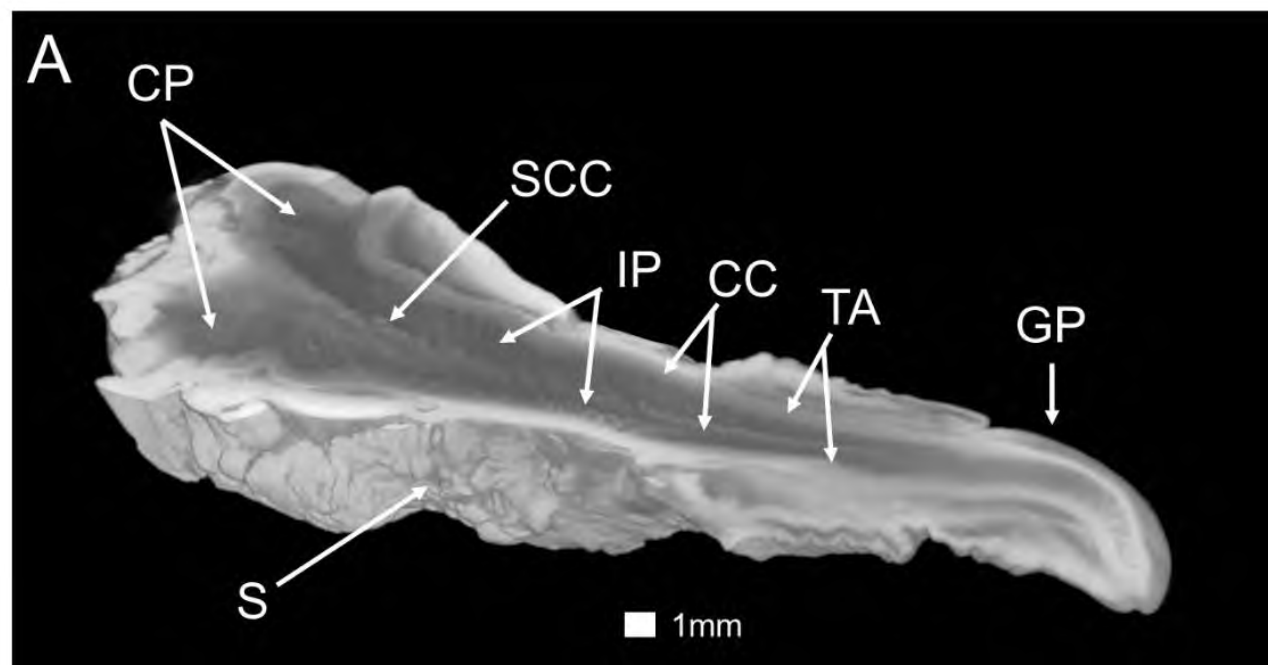




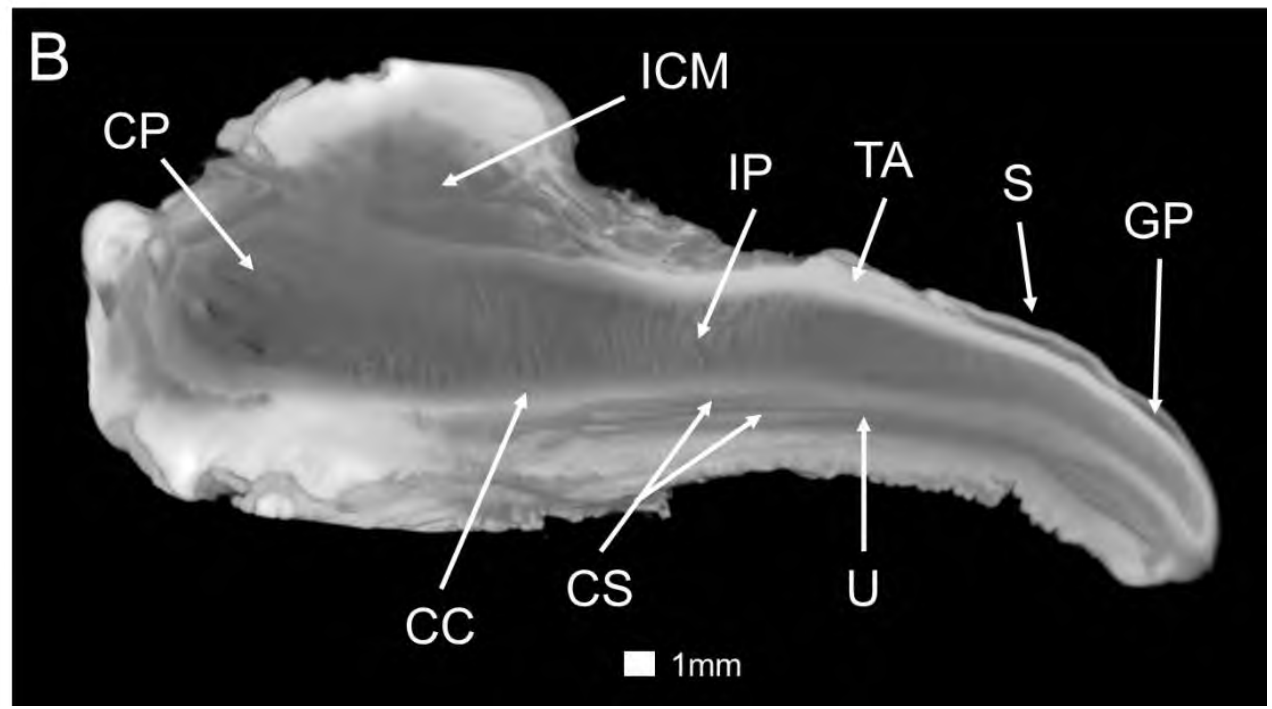

Fig. 3. Frontal (A) and sagittal (B) micro-CT section of laboratory rabbit penis. S - skin; ICM ischiocavernosus muscle; $\mathrm{CP}$ - crus penis; $\mathrm{GP}$ - glans penis; TA - tunica albuginea; $\mathrm{CC}$ - corpus cavernosum; SCC - septum of corpora cavernosa, IP - intracavernosal pillars; CS - corpus spongiosum; $\mathrm{U}-$ urethra.

\subsection{Histopathological imaging}

Multiple transversal and sagittal histopathological slides per penis sample were made. The histopathological images were compared with corresponding micro-CT sections. Almost the same amount of structures was identified in histological and micro-CT images. However, 2D histopathological slides stained by H\&E provides a view on cellular structures, such as urothelium and smooth muscle cells, which are not visible on micro-CT (voxel size was $-50 \mu \mathrm{m}$ ) (Fig. 4).
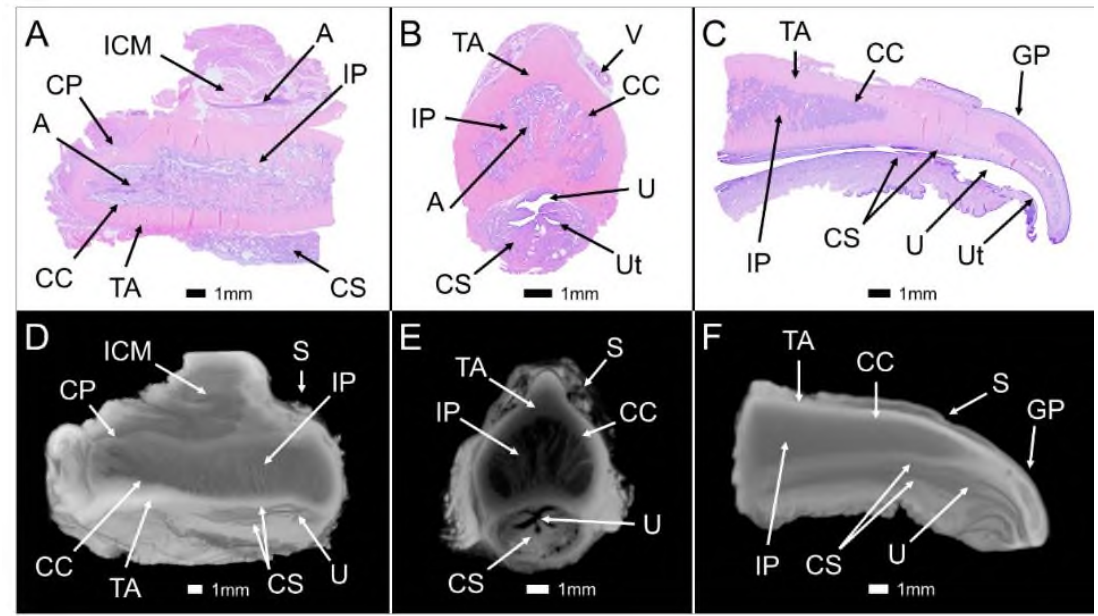

Fig. 4. Histopathological slides: basal sagittal (A), middle transversal (B), apical sagittal (C) and corresponding micro-CT sections (D-E). S - skin; ICM - ischiocavernosus muscle; $\mathrm{CP}$ - crus penis; 
GP - glans penis; TA - tunica albuginea; CC - corpus cavernosum; A - arteria; V - vein; SCC septum of corpora cavernosa; IP - intracavernosal pillars; CS - corpus spongiosum; U - urethra; Ut urothelium.

\section{Discussion}

The results of this study show capabilities to visualize surface and inner structures of laboratory rabbit penis by US, micro-CT, and histopathological analysis. Also, the degrees of detail of these methods of imaging are compared. The rabbits were chosen for this research because the penis size of this animal allows to use US, which is routine visualization methods used in clinical human medicine, along with technologically more complex methods, while rat does not give this possibility. Moreover, murine, and other animal models in pre-clinical trials are not very appropriate to use micro-CT because of the presence of a baculum. Rabbits are known to not to have this bone as like as in human [16]. That is why we suggest that this animal model is more appropriate, because we should not additionally focus on possible penile bone morphological alterations while assessing complex pathological changes, which are studied to be applied in clinical humane medicine.

US of rabbit penis give less detailed imaging as it is in clinical practice compared to other methods. However, it gives basis view to tunica albuginea and two corpora cavernosa, but only with an inserted urethral catheter. This gives possibilities to track the dynamics of rough alterations as in penile cancer and Peyronie's disease on a daily basis because US is non-invasive and non-destructive method [3,17]. This is the key advantage of penile US in animal model studies, which could reduce the need to use multiple cohorts of animals for several dates of morphological assessment of taken penile tissue. But it cannot provide quantitative analysis, such as morphometry in histopathological imaging and density analysis in micro-CT. We are sure that US should be used in animal model studies only as secondary and supportive visualization method.[18]

Micro-CT and histopathological analysis both require to take tissue sample from laboratory animal either by operation or by euthanasia. It is shown that micro-CT is able to give complex view on the whole penile sample due to $3 \mathrm{D}$ reconstruction. We are assured that micro-CT could be the key to revealing topical and dynamical morphological alterations in oncological, inflammatory, and fibrotic pathological responses. Basic histopathological analysis can provide only a $2 \mathrm{D}$ view on a limited part of the biological sample. There is developing methods of 3D histological reconstruction, but this method requires ideal sample preparation and complete absence of artefacts in slides. Moreover, in comparison with micro-CT 3D histological reconstruction is more time consuming for sample preparation, while for micro-CT one dehydration and staining procedure per sample is needed $[12,18]$. Therefore, we support the idea that micro-CT is the future for clinical and experimental pathology assessment [11].

The limitation of this study is that we only made a micro-CT study with acquisition voxel size parameter $-50 \mu \mathrm{m}$, which did not provide visualization of cellular structures such as smooth muscle cells and urothelium. However, it is known that with smaller voxel size enables micro-CT to visualize even cellular structures compared to histopathological analysis [11].

\section{Conclusion}

In summary, US could be used in the assessment of penile morphological alterations in a rabbit model as a secondary method, micro-CT provides nearly the same imaging quality 
compared to histopathological analysis. Moreover, it gives a complex view on taken tissue sample at once due to $3 \mathrm{D}$ reconstruction.

This work was funded by the grant of Foundation for Assistance to Small Innovative Enterprises no. 0059863. This agency had no role in study design, data collection and analysis, decision to publish, or preparation of the manuscript. Micro-CT was carried out in the Research and Education Center «Materials» of Don State Technical University (https://nano.donstu.ru/). Authors acknowledge the support of the Government of the Russian Federation (contract No. 075-15-2019-1880).

\section{References}

1. M. Vakulenko, N. Karnaukhov, V. Suhomlinov, N. Dobaeva, N. Akinina, A. Kochetkova, A. Ermakov, in E3S Web Conf., 135, 01075 (2019) doi: 10.1051/e3sconf/201913501075

2. M. N. Gurcan, L. E. Boucheron, A. Can, A. Madabhushi, N. M. Rajpoot, B. Yener, IEEE Rev. Biomed. Eng. 2, 147-171 (2009) doi: 10.1109/RBME.2009.2034865

3. F. Peyraud, C. Allenet, M. Gross-Goupil, C. Domblides, F. Lefort, A. Daste, M. Yacoub, T. Haaser, L. Ferretti, G. Robert, and A. Ravaud, Cancer Treat. Rev., 90, 102087 (2020) doi: 10.1016/j.ctrv.2020.102087

4. M. Bertolotto, I. Campo, C. Sachs, R. Ciabattoni, S. Bucci, M. Assunta, and S. Van Nieuwenhove, Abdom. Radiol., 45, $1973-1989$ (2020) doi: 10.1007/s00261-02002529-z

5. C. M. Lindquist, P. Nikolaidis, P. K. Mittal, and F. H. Miller, Abdom. Radiol. 455. K. M. Theisen, B. T. Kadow, P. J. Rusilko, Curr. Urol. Rep., 17, 54 (2016) doi: 10.1007/s11934-016-0616-0

6. C. M. Lindquist, P. Nikolaidis, P. K. Mittal, F. H. Miller, 2001-2017 (2020) doi: 10.1007/s00261-019-02301-y

7. M. C. Cho, W. H. Song, J. Paick, Sex. Med. Rev., 6, $572-582$ (2018) doi: 10.1016/j.sxmr.2018.02.007

8. M. Kogan, I. Popov, I. Popov, and S. Todorov, Urologiia, 4, 144-150 (2020) doi: 10.18565/urology.2020.4.144-150

9. M. I. Kogan, B. G. Amirbekov, M. N. Kryuchkova, V. V. Mitusov, V. V. Krasulin, D. V. Sizyakin, Urologiia, 5, 60-63 (2018) doi: 10.18565/urology.2018.5.60-63

10. P. Bidola, K. Morgan, M. Willner, A. Fehringer, S. Allner, F. Prade, F. Pfeiffer, K. Achterhold, J. Microsc., 266, 211-220 (2017) doi: 10.1111/jmi.12530

11. O. L. Katsamenis, M. Olding, J. A. Warner, D. S. Chatelet, M. G. Jones, G. Sgalla, B. Smit, O. J. Larkin, I. Haig, L. Richeldi, I. Sinclair, P. M. Lackie, and P. Schneider, Am. J. Pathol., 189, 1608-1620 (2019) doi: 10.1016/j.ajpath.2019.05.004

12. J. M. de S. e Silva, I. Zanette, P. B. Noël, M. B. Cardoso, M. A. Kimm, and F. Pfeiffer, Sci. Rep., 5, 1 (2015) doi: 10.1038/srep14088

13. P. Bankhead, M. B. Loughrey, J. A. Fernández, Y. Dombrowski, D. G. McArt, P. D. Dunne, S. McQuaid, R. T. Gray, L. J. Murray, H. G. Coleman, J. A. James, M. SaltoTellez, and P. W. Hamilton, Sci. Rep., 7, 16878 (2017) doi: 10.1038/s41598-01717204-5

14. F. Spani, M. P. Morigi, M. Bettuzzi, M. Scalici, and M. Carosi, PLoS One, 15, e0228131 (2020) doi: 10.1371/journal.pone.0228131

15. M. O’Neill, G. O. Huang, and D. J. Lamb, J. Sex. Med., 14, 1533-1539 (2017) doi: 10.1016/j.jsxm.2017.10.065 
16. J. Skonieczna, J. P. Madej, A. Kaczmarek- Pawelska, and R. Będziński, J. Vet. Med. Ser. C Anat. Histol. Embryol (2020) doi: 10.1111/ahe.12611

17. K. L. Sharma, M. Alom, L. Trost, Sex. Med. Rev., 8, 314-323 (2020) doi: 10.1016/j.sxmr.2019.06.004

18. J. Pichat, J. E. Iglesias, T. Yousry, S. Ourselin, M. Modat, Med. Image Anal., 46, 73105 (2018) doi: 10.1016/j.media.2018.02.004

19. M. Mazanko, E. Prazdnova, D. Rudoy, A. Ermakov, A. Olshevskaya, T. Maltseva, E3S Web of Conferences, 175, $01010 \quad$ (2020) INTERAGROMASH 2020 https://doi.org/10.1051/e3sconf/202017501010

20. A. A. Kotesova, S. V. Teplyakova, S. I. Popov, F. C. Kopylov, Ensuring assigned fatigue gamma percentage of the components. International Scientific Conference "Construction and Architecture: Theory and Practice of Innovative Development» (CATPID-2019): IOP Conference Series: Materials Science and Engineering, 698, 066029 (2019) doi:10.1088/1757-899X/698/6/066029 